\title{
Study of a Hollow Ultrasonic Rotary Motor
}

\author{
José M. Fernandez, Markus Flueckiger, and Yves Perriard, Senior Member IEEE \\ Ecole Polytechnique Fédérale de Lausanne (EPFL), Institut de Microtechnique (IMT) \\ Laboratoire d'Actionneurs Intégrés (LAI), Station 11, CH - 1015 Lausanne, Switzerland \\ Email : jose.fernandez@epfl.ch, markus.flueckiger@epfl.ch, yves.perriard@epfl.ch
}

\begin{abstract}
In this study, a hollow cylinder ultrasonic motor has been designed in order to be integrated into mechatronic devices where the bore is either occupied for functional reasons or must remain free. The design approach consists in performing a FE modelling of the motor structure. After finding the different vibration modes, it is possible to find out the axial and tangential deformation amplitudes of the resonator allowing the motor rotation when a pre-stressing force is applied to the rotor. Using an optimisation methodology based on a sensitivity analysis using in particular the design of experiments method and then a FE optimisation, it is possible to maximize the deformation amplitudes in both directions to obtain higher torque and speed of the motor. Along with this process, functional models are built in order to validate the adopted design methodology and verify if the chosen motor concept is appropriate.
\end{abstract}

\section{INTRODUCTION}

Hollow cylinder or ring type ultrasonic motors are integrated in industrial applications. However, drive electronics are rather complex, as for instance in the case of the travelling wave ultrasonic motor developed for autofocus lenses [1]. The idea is to use a single phase standing wave piezoelectric actuator dedicated to this application and to optimize it, based on a design methodology which combines concepts from design of experiments with numerical simulations using the ANSYS [2] finite element software. This type of motor presents some interesting characteristics compared to conventional electromagnetic motors. Indeed, energy consumption for a piezoelectric motor is roughly the same if it is blocked or runs free. Motor position is stable even when no power is applied and it provides high torque at low speeds.

The goal of this study is to research a hollow ultrasonic motor and to improve its characteristics, particularly torque and rotation speed, by optimising the dimension of the different components. First of all, the construction of a numerical model of a dedicated actuator will be accomplished. Thanks to a sensitivity analysis based on design of experiments [3], the significant parameters of the actuator have been obtained and its optimisation using the finite element model has been carried out. Next section presents the different results obtained. Along with this process, functional models have been realized and tested in order to validate the adopted design methodology.

\section{FE Modelling ANd Simulations}

\section{A. Introduction}

A standing wave type ultrasonic motor has been chosen for this specific application. Indeed, the simplicity of the geometry and the way to drive the motor are some advantages in comparison to the travelling wave or step by step ultrasonic motor. Then, a motor structure has to be designed and FE modelling is used to find out the different resonance modes of the stator. Simulations have been carried out and deformation amplitudes have been obtained.

\section{B. Standing wave type ultrasonic motor}

1) Structure: The stator consists of a piezoelectric hollow cylinder. Aluminum pushers are glued along the end of the cylinder to operate the contact with the rotor. The cylinder is polarized radially. A common drain covers the entire inside surface and two separate groups cover the outside surface. These electrode groups permit excitation of the stator in a special eigenmode (coupled tangential axial mode) in which the pushers move back and forth at an angle to the end of the cylinder. The number of electrodes in each group is equal to the number of wavelengths around the circumference of the cylinder. Excitation of the required oscillation mode involves placing a single-phase sine wave voltage on one of the electrode groups. The other group is allowed to float. The rotor is pressed against the pushers by means of a pre-stressing force (spring). Then, due to the oscillations of the stator when excited at the resonant frequency, the pushers impart microimpulses to the rotor which make it rotate. Rotation in the opposite direction can be obtained by interchanging the roles of the electrode groups. Figure 1 shows the arrangement of
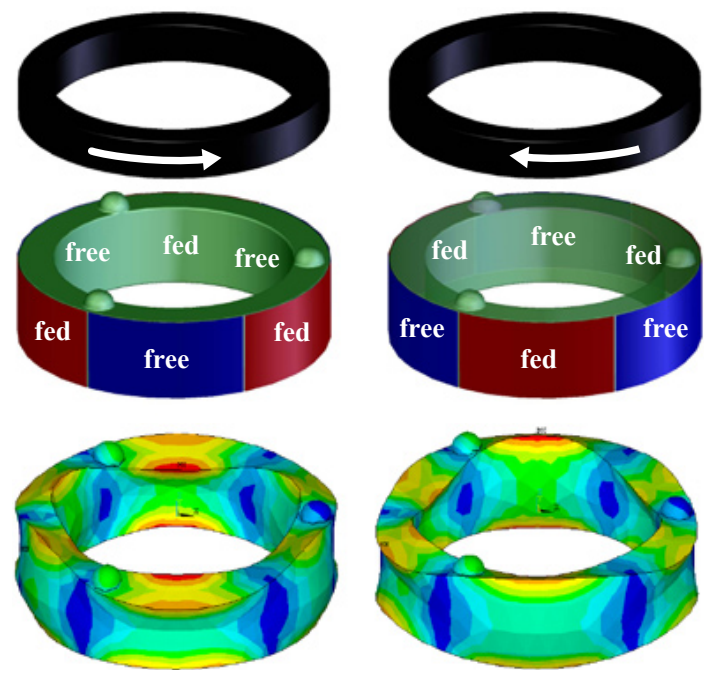

Figure 1. Stator structure and excitation for counterclockwise and clockwise rotation respectively 
the pushers, electrode groups and the resulting deformation of the cylinder for excitation of the $3^{r d}$ tangential-axial mode in the stator [4].

2) Dimensions and materials: Four different geometries of the stator structure have been simulated. Figures 2 and 3 as well as Table I give the different dimensions of the four piezoelectric ceramics that have been used. These values have been taken into account for the simulations even if the pushers will be modeled as half spheres for simplifications reasons.

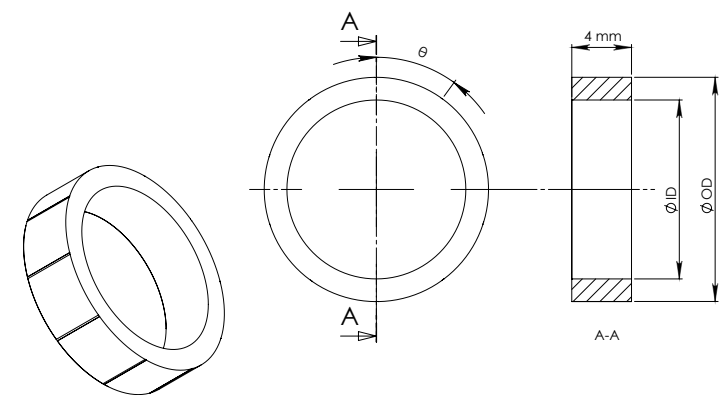

Figure 2. Dimensions of the piezoelectric ceramic ring
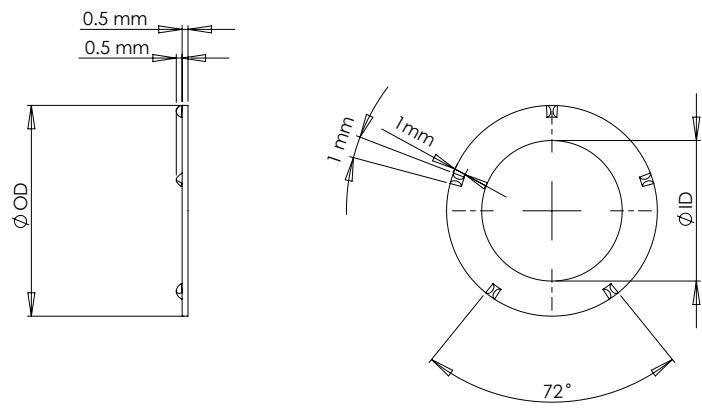

Figure 3. Ring with 5 pushers for the contact

Table I

DIMENSIONS OF THE FOUR PIEZOELECTRIC CERAMICS

\begin{tabular}{|c|c|c|c|c|}
\hline & $\varnothing$ OD & $\varnothing$ ID & Sectors/Pushers & $\theta$ \\
\hline \hline Ceramic $n^{\circ} 1$ & $18 \mathrm{~mm}$ & $12 \mathrm{~mm}$ & $10 / 5$ & $36^{\circ}$ \\
\hline Ceramic $n^{\circ} 2$ & $18 \mathrm{~mm}$ & $12 \mathrm{~mm}$ & $6 / 3$ & $60^{\circ}$ \\
\hline Ceramic $n^{\circ} 3$ & $15 \mathrm{~mm}$ & $12 \mathrm{~mm}$ & $10 / 5$ & $36^{\circ}$ \\
\hline Ceramic $n^{\circ} 4$ & $15 \mathrm{~mm}$ & $12 \mathrm{~mm}$ & $6 / 3$ & $60^{\circ}$ \\
\hline
\end{tabular}

The materials are a hard PZT (from EBL [5]) for the piezoelectric hollow cylinder (with Nickel electrodes) and aluminium for the pushers. The properties of the piezoelectric ceramic are defined in the Appendix.

\section{FE Simulations}

1) Introduction: First of all, a modal analysis has been carried out to find out the different vibration modes of the stator (composed of the piezoelectric ceramic and the contact ring). This has been done for the four different resonator configurations. Then, thanks to the modal analysis, it has been possible to determine the resonant frequency of each structure by performing an harmonic simulation. Finally, the transient response has been calculated for each case. A sinusoidal AC voltage of $100 \mathrm{~V}$ (RMS) has been applied to the set of active electrodes of the piezoelectric cylinder. For the simulations, a damping coefficient (adapted for each structure) has been taken into account.

2) Results: Different simulations have been carried out for each structure. The following results are those obtained for a stator composed of ceramic $n^{\circ} 4$. Figure 4 shows the $3^{\text {rd }}$ vibration mode for a frequency of $411 \mathrm{kHz}$. Then, Fig. 5 and Fig. 6 depict the motion of a point at the surface of the pusher: the graphs show the amplitude profiles of the axial and tangential oscillations respectively. Finally Fig. 7 depicts the typical trajectory of a pusher surface point.
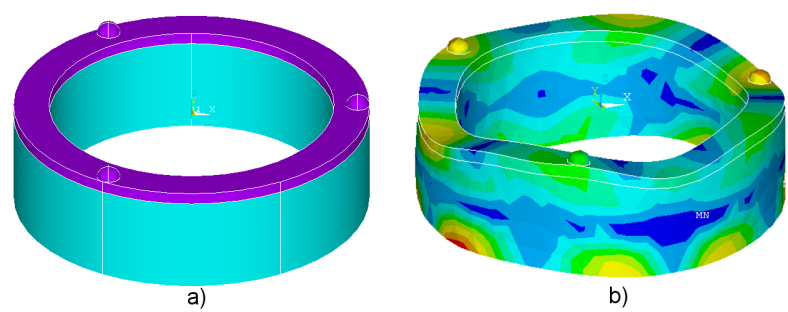

b)

Figure 4. a) FE model, b) Vibration mode at the resonant frequency $(411 \mathrm{kHz})$

After performing the simulations for the four different stator configurations, it is then possible to compare the structures. Table II sums up the different results obtained.

Table II

COMPARISON OF THE DIFFERENT STATOR STRUCTURES

\begin{tabular}{|c|c|c|}
\hline Actuator & Resonance freq. & Amplitudes (axial/tang.) \\
\hline \hline$n^{\circ} 1$ & $f_{\text {res }}=410.09 k H z$ & $0.062 \mu \mathrm{m} / 0.016 \mu \mathrm{m}$ \\
\hline$n^{\circ} 2$ & $f_{\text {res }}=388.11 k H z$ & $0.057 \mu \mathrm{m} / 0.078 \mu \mathrm{m}$ \\
\hline$n^{\circ} 3$ & $f_{\text {res }}=462.10 k H z$ & $0.059 \mu \mathrm{m} / 0.038 \mu \mathrm{m}$ \\
\hline$n^{\circ} 4$ & $f_{\text {res }}=411.23 k H z$ & $0.16 \mu \mathrm{m} / 0.22 \mu \mathrm{m}$ \\
\hline
\end{tabular}

3) Discussion: As it can be seen, the stator composed of ceramic $n^{\circ} 4$ gives the maximum deformation amplitudes in axial and tangential directions. Furthermore, the resonant frequencies are quite the same for all the structures, being around $400 \mathrm{kHz}$. To definitively confirm the simulations, measurements of the axial and tangential displacements need to be done using in particular an interferometer. Next section presents some measurements done to validate the resonant frequency of the different structures found numerically by using an impedance analyzer. Then, simulations and measurements are compared to validate the design methodology.

\section{Measurements And validation}

\section{A. Prototyping and measurements}

After the realization of several functional models (see Fig. 8 for the case of an actuator composed of ceramic $n^{\circ} 3$ ), tests 


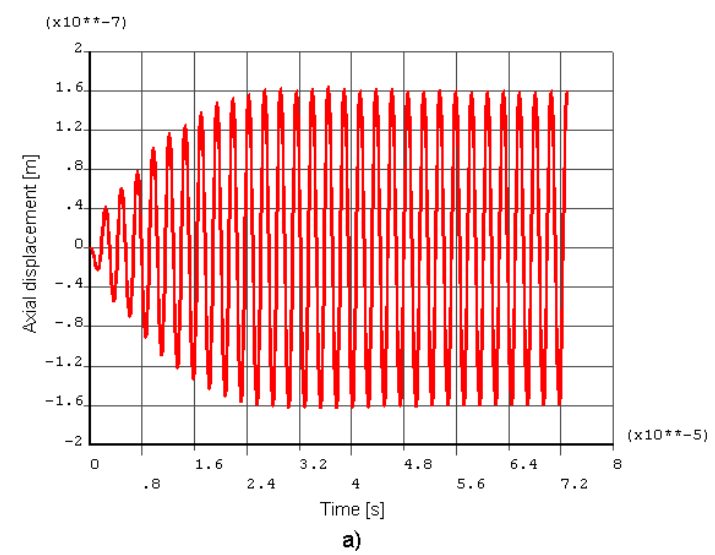

Figure 5. a) Axial displacement of a pusher surface point

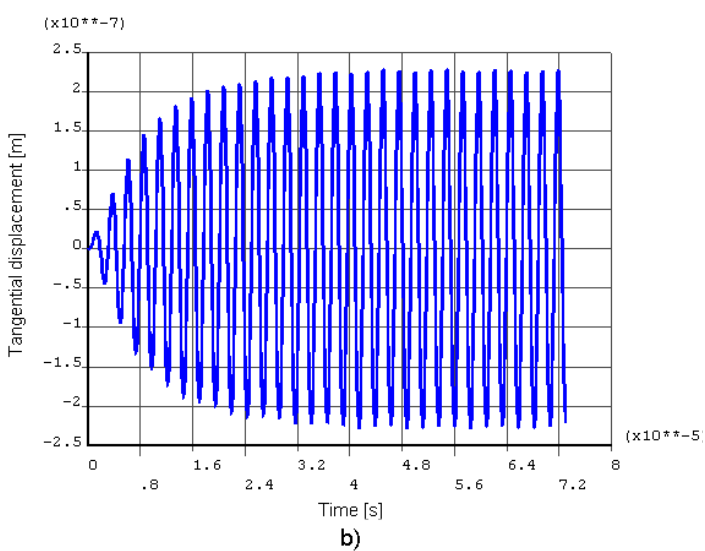

Figure 6. a) Tangential displacement of a pusher surface point

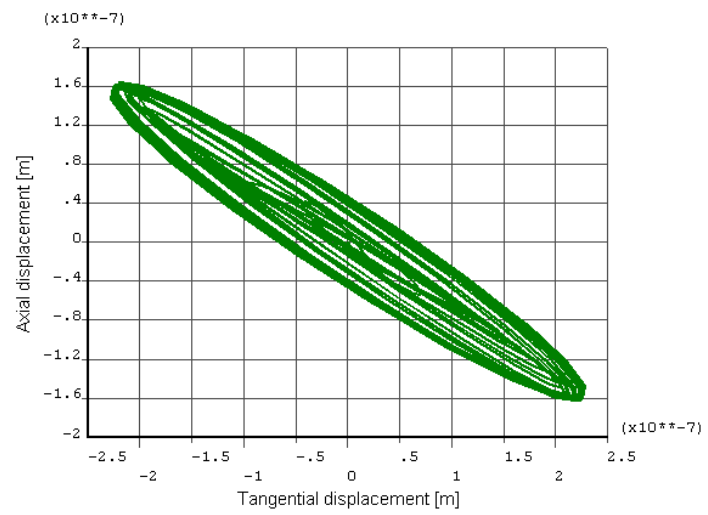

Figure 7. Elliptical displacement of a point at the surface of the pusher

have been realized for each structure. The first measurements have been carried out using an impedance analyzer to observe the different vibration modes and determine where is located the resonant frequency. Figure 9 presents the admittance curve observed for the case of an actuator composed of ceramic $n^{\circ} 4$. This has also been done with a series of 10 or 20 samples of each structure to prove a certain repeatability of the results found.

Then, with the aim of comparing directly the deformation amplitudes found numerically, measurements have been done using an interferometer to obtain the axial and tangential displacements of a pusher surface point. Figure 10 shows this comparison for the axial deformation amplitude. These results are discussed in the next section.

\section{B. Validation}

First of all, Table III shows the comparison between the resonant frequencies found through simulation for each structure and the corresponding mode found experimentally. As it can be seen, values are quite the same for structures composed of ceramic $n^{\circ} 1$ and $n^{\circ} 4$ and less than 5\% different for structures composed of ceramic $n^{\circ} 2$ and $n^{\circ} 3$.

Table III

RESONANT FREQUENCIES COMPARISON

\begin{tabular}{|c|c|c|}
\hline Actuator & Simulated res. freq. & Experimental res. freq. \\
\hline \hline$n^{\circ} 1$ & $f_{\text {res }}=410.09 \mathrm{kHz}$ & $f_{\text {res }}^{\prime}=410.5 \mathrm{kHz}$ \\
\hline$n^{\circ} 2$ & $f_{\text {res }}=388.11 \mathrm{kHz}$ & $f_{\text {res }}^{\prime}=406.0 \mathrm{kHz}$ \\
\hline$n^{\circ} 3$ & $f_{\text {res }}=462.10 \mathrm{kHz}$ & $f_{\text {res }}^{\prime}=440.63 \mathrm{kHz}$ \\
\hline$n^{\circ} 4$ & $f_{\text {res }}=411.23 \mathrm{kHz}$ & $f_{\text {res }}^{\prime}=412.0 \mathrm{kHz}$ \\
\hline
\end{tabular}

Furthermore, Fig. 10 shows that the axial deformation amplitudes found experimentally and through simulation are quite similar. Some differences are still present but they can be explained by the fact that the damping coefficient can still be adapted in the numerical model and the frequency shift is due to the measurements errors. Nevertheless, numerical modelling could be validated, in particular by comparing the results found for the other actuator structures. Indeed, from the simulation results it can not be concluded that the rotor will move when pressed against the stator. As the speed and torque of the rotor are hypothetically directly proportional to the axial and tangential displacements of the friction points (pushers), the amplitudes should be sufficiently high to make the rotor move. At the moment, some experiments have been done by trying to put into contact an annular ring (slightly prestressed) with the stator (fed by an $\mathrm{AC}$ voltage at the resonant frequency of the corresponding stator structure). Motion of this annular ring could be observed, especially using actuators $n^{\circ} 1$ and $n^{\circ} 2$, but investigation should go on. In this way, several points have to be taken into account in the future work:

- The applied voltage should be high enough to obtain deformation amplitudes allowing to make the rotor rotate.

- The ceramic has to be fed at its precise resonant frequency, which would change with heating and need to be controlled through appropriate electronics.

- The choice of the glue and its process has to be done carefully because contact points (pushers) must fit very narrow tolerances.

- The pre-stressing force which influences speed and torque of the motor should be determined precisely by carrying a contact simulation and also experimental measurements. 


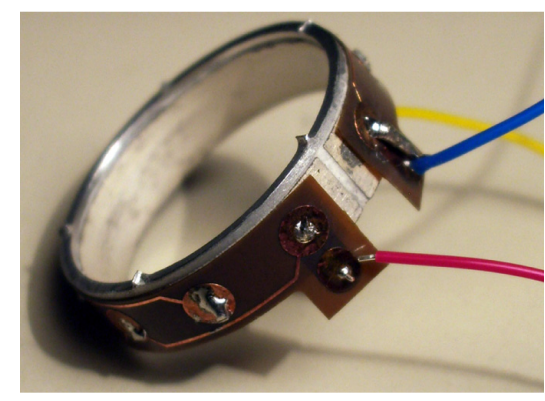

Figure 8. Functional model of a $5^{\text {th }}$ mode stator
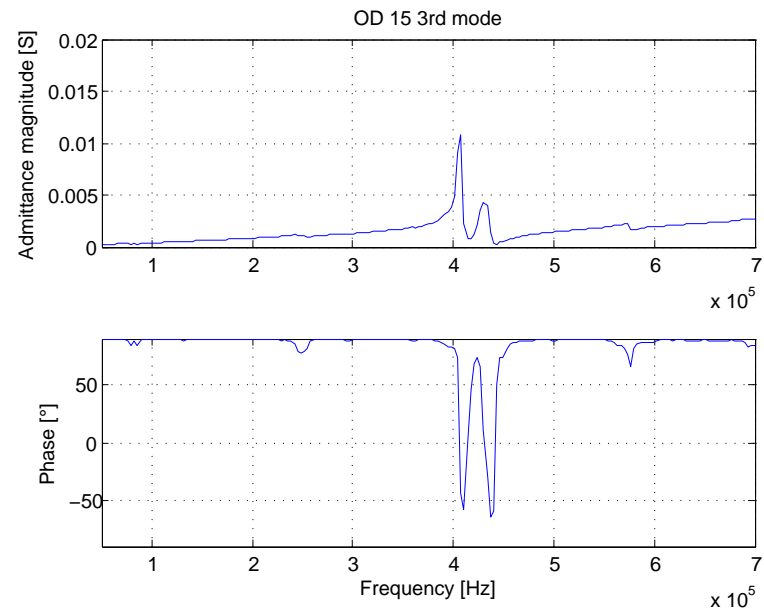

Figure 9. Admittance amplitude and phase of actuator $n^{\circ} 4$

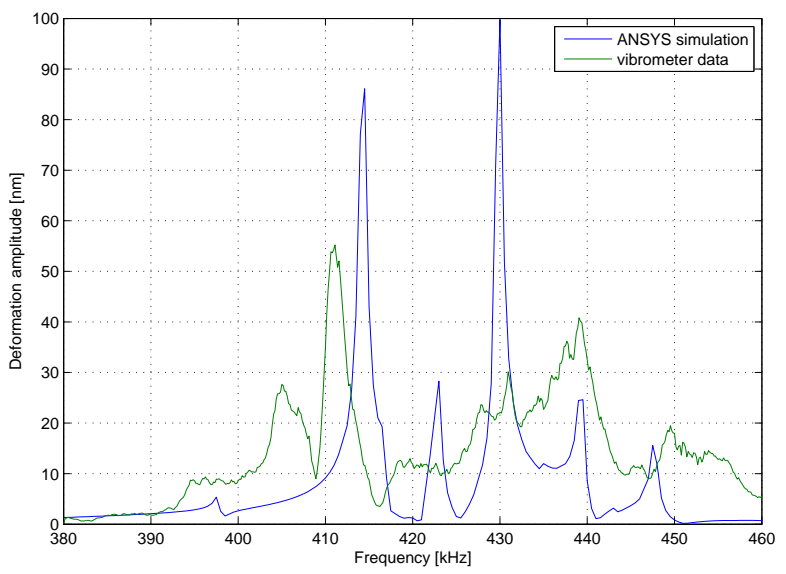

Figure 10. Comparison of simulated and measured axial deformation amplitude of actuator $n^{\circ} 4$

\section{CONCLUSION}

The study of a standing wave type ultrasonic rotary motor has been presented. After choosing a design for the actuator, a numerical model has been built. FE simulation results have shown that the maximal deformation amplitudes are found using a resonator with a third vibration mode at a resonant frequency of $411 \mathrm{kHz}$. Axial and tangential displacements are about $0.16 \mu \mathrm{m}$ and $0.22 \mu \mathrm{m}$, respectively. Elliptical motions of the pushers surface points have also been observed. In parallel, some functional models have been built and tested. The admittance response of each stator structure has been measured and also the deformation amplitudes have been measured with a laser interferometer. By comparing the simulations results with the experimental data obtained, it could be noticed that the results are similar and the design methodology could be validated.

Nevertheless, further developments are needed at this stage of the study. Indeed, the contact problems between the resonator and the rotor should be carefully investigated. FE simulations should give more information about the relationship between deformation amplitudes and torque or speed of the motor. Moreover, more practical experiments need to be carried out in order to verify if the concept of a standing wave type ultrasonic motor is judicious to fulfil the different requirements of hollow bore devices.

\section{APPENDIX}

Material properties of the used PZT ceramic from EBL [5] company.

$$
\text { Density: } \rho=7600 \mathrm{~kg} / \mathrm{m}^{3} \text {. }
$$

Stiffness matrix:

$$
c^{E}=\left[\begin{array}{cccccc}
14.7 & 8.11 & 8.11 & 0 & 0 & 0 \\
& 14.7 & 8.11 & 0 & 0 & 0 \\
& & 13.2 & 0 & 0 & 0 \\
& & & 3.13 & 0 & 0 \\
& & & & 3.13 & 0 \\
& & & & & 3.29
\end{array}\right] \cdot 10^{10} \mathrm{~N} / \mathrm{m}^{2}
$$

Piezoelectric constants:

$e=\left[\begin{array}{cccccc}0 & 0 & 0 & 0 & 10.34 & 0 \\ 0 & 0 & 0 & 10.34 & 0 & 0 \\ -3.87 & -3.87 & 13.91 & 0 & 0 & 0\end{array}\right] \quad A s / m^{2}$

Dielectric constants:

$$
\epsilon^{T}=\left[\begin{array}{ccc}
1.14 & 0 & 0 \\
0 & 1.14 & 0 \\
0 & 0 & 0.89
\end{array}\right] \cdot 10^{-8} \mathrm{As} / \mathrm{Vm}
$$

[1] I.Okumura and H.Mukohjima, A Structure of Ultrasonic Motor for Autofocus Lenses, Proceeding Motor-Con'87,1987, pp. 75

[2] Ansys Multiphysics 8.1 version, Ansys Inc., 2004.

[3] J. Fernandez and Y. Perriard, Sensitivity Analysis and Optimization of a Standing Wave Ultrasonic Linear Motor, IEEE Transactions on Ultrasonics, Ferroelectrics and Frequency Control, vol. 53, n 7 , pp. 13521361, 2006.

[4] O. Vyshnevskyy, S. Kovalev, W. Wischnewskiy, New type of piezoelectric standing wave ultrasonic motors with cylindrical actuators, ACTUATOR 2004, 9th International Conference on New Actuators, Germany, 2004.

[5] EBL Piezoelectric Precision, www.eblproducts.com. 